\title{
Síndrome de Allen Master: causa excepcional de abdomen agudo en el embarazo
}

\author{
Cristina Álvarez C. ${ }^{1,2}$, Victoria Pascual E. ${ }^{1}$, Laura Barrero R. ${ }^{1,2}$, Blanca Heras P. ${ }^{1}$, \\ Derek Tami G. 1 \\ ${ }^{1}$ Servicio de Obstetricia y Ginecología. Hospital Clínico Universitario de Valladolid. ${ }^{2}$ Facultad de Medicina de la \\ Universidad de Valladolid. España.
}

\section{RESUMEN}

Se presenta un caso de abdomen agudo en una gestante de 20 semanas, por una torsión anexial. Ante la ausencia de signos ecográficos compatibles con patología en los primeros momentos y el empeoramiento clínico, se solicitó una RMN que permitió la aproximación diagnóstica y facilitó la indicación urgente de cirugía abdominal. En el curso de la laparotomía se diagnosticó una hernia interna del anexo, con marcado componente vascular a través de un defecto del ligamento ancho, que obligó a la anexectomía. Tras el diagnóstico y el tratamiento realizado, la gestación continúa con buenos resultados materno-fetal. Estos orificios han sido presentados excepcionalmente como causa de hernias internas digestivas, urológicas y anexiales, siendo controvertido su origen, congénito, inflamatorio o traumático. Se repasan las pruebas complementarias indicadas en el manejo diagnóstico del dolor abdominal en el embarazo, incluyendo las últimas recomendaciones de la European Society of Urogenital Radiology-ESUR.

\section{PALABRAS CLAVE: Abdomen agudo, síndrome de Allen-Masters, ligamento ancho, embarazo}

\section{SUMMARY}

A case of acute abdomen in a pregnant 20 weeks is presented with an adnexal torsion. In the absence of pathologic ultrasound signs with in the early stages and clinical worsening, was requested an MRI that allowed the diagnostic approach and the indication for urgent abdominal surgery. During laparotomy was observed an internal adnexal hernia through a defect in the broad ligament, with marked vascular component which forced the adnexectomy. After the diagnosis and treatment performed, the pregnancy continues with good maternal and fetal outcomes. These defects were presented exceptionally as a cause of digestive, urological and adnexal internal hernias. The origin remains controversial, congenital, inflammatory or traumatic. Complementary tests in the diagnostic management of abdominal pain in pregnancy are reviewed, including the latest recommendations of the European Society of Urogenital Radiology-ESUR.

\section{KEY WORDS: Acute abdomen, Allen-Masters syndrome, broad ligament, pregnancy}

\section{INTRODUCCIÓN}

La aproximación diagnóstica y terapéutica al dolor abdominal durante la gestación sigue siendo un reto para el clínico: la sintomatología se con- funde con las modificaciones fisiogravídicas, hay limitaciones en la indicación e interpretación de las pruebas complementarias, que ocasionan retardo en el diagnóstico y tratamiento que puede influir negativamente en el pronóstico materno y fetal. 
El síndrome de Allen-Masters se diagnostica por la presencia de dolor pélvico crónico asociado a la hipermovilidad cervical, retroversión y defectos del ligamento ancho, percibidos en general en el curso de una laparotomía o laparoscopia. Estas lesiones, han sido comunicadas como causa de hernias internas intestinales y de forma excepcional del anexo, siendo anecdótica la publicación de casos en la literatura.

La confirmación de este hallazgo en una gestante diagnosticada de abdomen agudo, justifica su comunicación, dada la complejidad de su aproximación diagnóstica y la imposibilidad de la confirmación prequirúrgica.

\section{Caso clínico}

Primigesta de 29 años, sin antecedentes de importancia, que consultó a las 20 semanas de gestación por dolor abdominal. En sus antecedentes ginecológicos presenta ciclo menstrual normal, dismenorrea habitual y dispareunia profunda. Refiere dolor cólico en fosa ilíaca izquierda (FII), de inicio brusco, progresivo, irradiado a región inguinal y pierna izquierda, con náuseas y vómitos alimenticios, sin signos de alarma obstétrica. Está afebril, postrada, con abdomen blando, depresible, y dolor a la palpación profunda en FII, sin signos de irritación peritoneal. El tono uterino es normal con fondo 2 traveses sobre ombligo. El cuello está formado y cerrado, y el tacto vaginal despierta intenso dolor en fondo de saco izquierdo.

Con la ecografía se confirmó la vitalidad fetal, con biometría acorde a la edad gestacional, placenta anterior normoinserta, cervicometría normal, sin observarse en ese momento imágenes sospechosas de patología anexial, ni líquido libre en cavidad peritoneal.

Ante la persistencia del dolor se decidió ingreso de la paciente para observación, control sintomático y solicitud de otras pruebas complementarias. La ecografía abdomino-pélvica e inguinal no mostró alteraciones digestivas, urológicas, ni defectos de la pared abdominal y confirmó la normalidad de los vasos pélvicos e inguinales.

Doce horas después, comienza súbitamente nuevo episodio doloroso de características similares. Se solicitó una resonancia magnética nuclear (RMN), que informó de una imagen nodular, con componente quístico heterogéneo en secuencias $\mathrm{T} 2$, de $53 \times 40 \times 31 \mathrm{~mm}$, a nivel parauterino izquierdo por detrás de la vejiga, que podría corresponder a una masa anexial, asociado a mínima cantidad de líquido libre en la pelvis (Figura 1). Se realizó nueva ecografía endovaginal, observando formación heterogénea parauterina izquierda de 57,7 x $57,9 \mathrm{~mm}$, con mapa color negativo (Figura 2). En la analítica se evidenció un incremento de la leucocitosis.
Ante la sospecha de torsión anexial, se decidió laparotomía. Durante la intervención se observó el anexo adyacente a la vejiga, alojado en la plica vesicouterina, tras haber atravesado el ligamento ancho por orificios de unos $4 \mathrm{~cm}$ en las dos caras del mismo (Figura 3). El pedículo vascular estaba torsionado y el anexo presentaba signos macroscópicos de necrosis (Figura 4). Se procedió a la reducción de la hernia, la anexectomía y la reparación del defecto en el ligamento ancho.

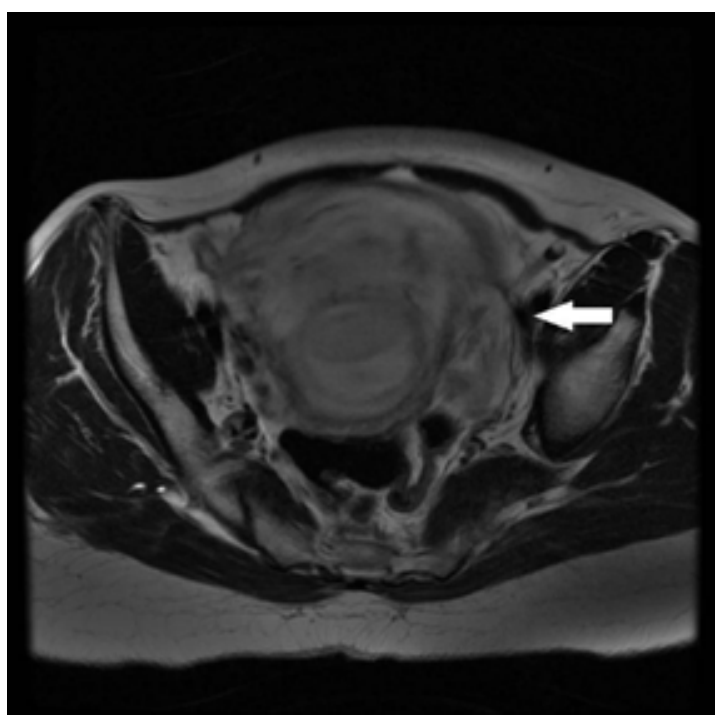

Figura 1. Imagen de RMN en secuencia T2. Se observa formación nodular con componente quístico heterogéneo, en zona parauterina izquierda por detrás de la vejiga, compatible con formación anexial.

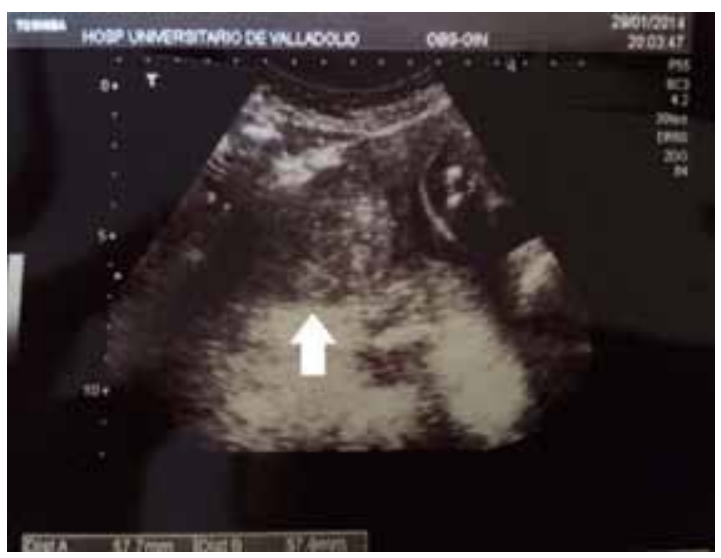

Figura 2. Imagen de ecografía endovaginal, en la que se observó una formación heterogénea parauterina izquierda de 57,7 x 57,9 mm, con mapa color negativo. 


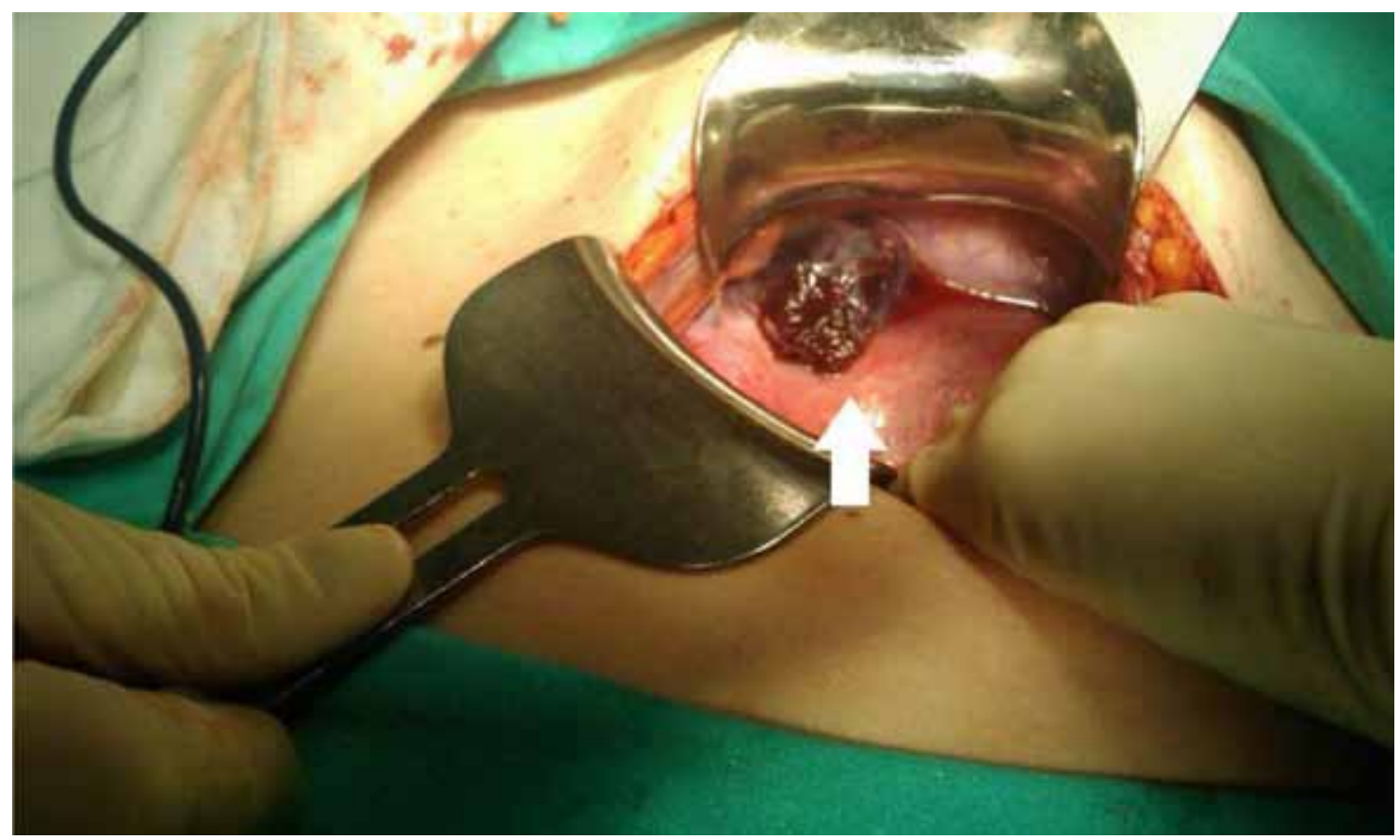

Figura 3. Hallazgos durante la laparotomía. Masa quística con aspecto isquémico localizado en el lado izquierdo en la plica vesicuterina, entre cara posterior de la vejiga y cara anterior del útero.

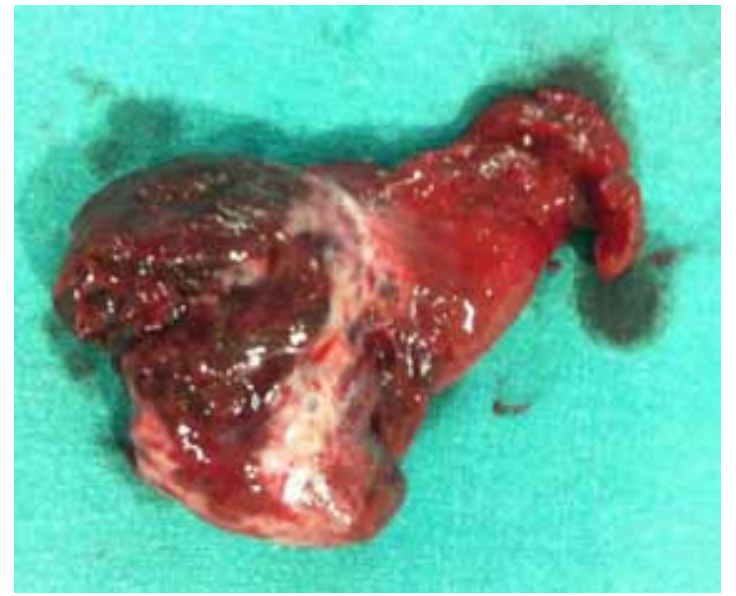

Figura 4. Imagen macroscópica de la pieza quirúrgica de anexectomía izquierda.

La evolución postoperatoria fue favorable. El estudio macroscópico de la pieza quirúrgica, muestra un ovario y trompa congestivos. El estudio microscópico, evidencia vasos de mediano calibre trombosados, con depósitos de fibrina en su pared y áreas extensas de hemorragia, compatible con un infarto agudo ovárico hemorrágico.
La evolución de la gestación fue normal hasta el inicio espontáneo del parto a las $39+3$ semanas. Se obtuvo un recién nacido femenino de 3500 gramos, con Apgar y $\mathrm{pH}$ umbilical normal.

\section{DISCUSIÓN}

El caso presentado corresponde a un hernia interna del anexo izquierdo a través de un orificio del ligamento ancho, que se diagnosticó durante el acto quirúrgico indicado por la presencia de abdomen agudo en un gestante de 20 semanas.

Los defectos del ligamento ancho se han identificado como causa de hernias internas de forma aislada desde finales del siglo XIX. Correspondían a hallazgo en necropsias, como el comunicado por Quain en 1861 (una mujer fallecida por una obstrucción intestinal que presentaba un asa estrangulada en un orificio del ligamento ancho derecho). Posteriormente se publicaron otros casos tras laparotomías por sospecha de obstrucción intestinal en mujeres de todas las edades. En 1920 Richardson relacionó por primera vez estos casos excepcionales con el antecedente de la operación de BaldyWebster (1).

Hunt planteó tres hipótesis etiopatogénicas para esos defectos: el origen congénito, las secuelas de un proceso inflamatorio o el traumatismo directo (2). Además, realizó descripciones pormenorizadas 
de sus localizaciones y las clasificó según el aspecto macroscópico: tipo "pouches" o defecto en bolsa (afecta a una sola hoja del ligamento), y "fenestrae", con orificio en ambas hojas. El autor, tras revisión de los 18 casos publicados hasta entonces, en multíparas entre 30 y 70 años, planteó la hipótesis que durante la gestación el orificio aumentaba por la presión intraabdominal o la distención del sistema de suspensión uterina, y que la pérdida de la elasticidad de los tejidos por la edad, estrechando el orificio, era responsable de la aparición de la clínica (2).

Se ha relacionado el tipo macroscópico del defecto con la clínica. El tipo "pouche" puede presentarse como dolor crónico sin afectación vascular, mientras que el tipo "fenestrae" se asocia más frecuentemente a hernia con isquemia intestinal $(3,4)$. Nuestro caso corresponde, según esta clasificación, a un defecto tipo fenestrae localizado entre el mesosalpinx y la base del ligamento ancho.

Los defectos peritoneales que afectan al sistema de soporte uterino (ligamentos anchos, ligamento cardinal de Mackenrodt y ligamentos uterosacros) junto con la presencia de hipermovilidad uterina, retroversión forzada, dismenorrea y dispareunia profunda, fueron recogidos bajo el epígrafe de "universal-joint cervix" y posteriormente con el nombre de síndrome de Allen-Masters. Estos autores describieron estos hallazgos en 28 mujeres sometidas a laparotomía por dolor pélvico (5). Lo asociaron a traumatismos en las relaciones sexuales o a partos difíciles (fórceps altos), precipitados o de fetos macrosómicos. La exploración pélvica confirmó el defecto en el sistema de soporte del útero en los 28 casos, siendo en 24 bilaterales. La reparación de estos defectos, junto con otros actos quirúrgicos adicionales, como apendicectomía, o colposuspensión mediante plicatura de ligamentos útero-sacros, mejoró la clínica de la gran mayoría (5). Nuestra paciente presentaba dismenorrea habitual y dispareunia profunda, síntomas del síndrome de Allen-Masters, si bien no tenía ninguno de los factores de riesgo definidos por los autores.

Se ha considerado el origen congénito de los defectos relacionándolos con la rotura de formaciones quísticas que se localizarían en el peritoneo y que corresponderían a reductos embrionarios de los conductos de Müller y de Wölf (6). Más recientemente, estos defectos peritoneales han sido relacionados con la endometriosis al observarse en laparoscopias diagnósticas por dolor pélvico; aunque en muchas no se encuentran todos los hallazgos recogidos en la descripción del síndrome, ni los factores de riesgo definidos clásicamente $(7,8)$. Batt insiste, en cambio, en el origen traumático de las lesiones y la ausencia de relación con la endometriosis (9). Nosotros no observamos lesiones compatibles con endometriosis, ni se identificaron factores traumáticos por lo que podemos pensar en un origen congénito del defecto.

Del total de las hernias internas documentadas, sólo un $4-7 \%$ son secundarias a un defecto del ligamento ancho (10). Habitualmente es el tubo digestivo el órgano comprometido, siendo la más frecuente la hernia de sigma o yeyuno (10-15), y excepcional la afectación de la vía urinaria (16) o el ovario y la trompa (4). Nuestra paciente puede considerarse un caso excepcional, puesto que solo se han podido encontrar en la literatura publicada otros tres casos en los que el órgano herniado a través del orificio es el anexo $(4,17,18)$, ninguno durante el embarazo.

El diagnóstico preoperatorio es muy difícil. La aproximación se basa en dolor abdominal tipo cólico asociado a síntomas digestivos, junto a los hallazgos de las pruebas complementarias (ecografía, tomografía computarizada, RMN y laparoscopia). Las dificultades se incrementan si el cuadro afecta una gestante, en el que estas pruebas y su interpretación están limitadas.

Tal y como recogen las recientes recomendaciones de la European Society of Urogenital Radiology-ESUR para la aproximación diagnóstica del dolor abdominal en el embarazo, deben ser evitadas en principio las exploraciones ionizantes. Los ultrasonidos son la exploración preferida en el curso de la gestación, por vía abdominal, pélvica y vaginal. La RMN puede ayudar cuando los ultrasonidos no sean conclusivos, reservando la tomografía a bajas dosis para aquellas situaciones en los que no sea posible la realización de una RMN (19). En el caso presentado y tras el empeoramiento clínico, se optó por el estudio de imagen mediante RMN. Con esta prueba se sospechó el accidente anexial, si bien no se pudo aproximar el diagnóstico a la hernia interna en el defecto del ligamento ancho.

No se han documentado efectos adversos de los ultrasonidos durante la gestación. Aunque existe la recomendación de utilizar como límite intensidades menores de $720 \mathrm{~mW} / \mathrm{cm}^{2}$ y limitar el uso del Doppler al tiempo mínimo necesario (20). Tampoco se han demostrado efectos secundarios graves sobre el feto con las imágenes obtenidas por RMN $\mathrm{T} 1$, y se recomienda el uso de esta técnica siempre que se vayan a obtener datos clínicos importantes que solo fueran posibles utilizando radiaciones ionizantes. En cambio no se ha podido demostrar aún la seguridad en 3T. No se cree, tampoco, que exista estímulo acústico ni incremento de temperatura suficiente para provocar lesiones fetales en el curso de la exploración (19), aunque si se recomienda evitar el uso de gadolinio por su paso transplacentario y su potencial relación con la fibrosis renal fetal.

Se optó por la reducción de la herniación ovárica a través del defecto. Se realizó una anexectomía 
al presentar el anexo signos de necrosis, finalizando con la sutura del defecto en ambas capas del ligamento. La mayoría de los autores recomiendan este tratamiento, bien mediante laparotomía o laparoscopia (15). Gupta y cols proponen la sección del ligamento redondo homolateral para ampliar el defecto y evitar la recidiva (13).

\section{CONCLUSIÓN}

El abordaje del dolor abdominal en la gestante sigue siendo un reto en la clínica diaria. No debemos despreciar la utilidad de pruebas de imagen como la RMN, en aquellos casos en los que, estando disponible, los ultrasonidos no sean conclusivos con el objetivo de no retardar el diagnóstico y el tratamiento adecuado. Las hernias internas son excepcionales, más si afectan al anexo, y el diagnóstico preoperatorio de un defecto del ligamento ancho sigue pareciendo en este momento casi imposible.

\section{REFERENCIAS}

1. Richardson EP. Intestinal obstruction following BaldyWebster operation for retroversion: report of case. Surg Gynecol Obstet 1920;XXXI:90.

2. Hunt A. Fenestra and pouches in the broad ligament as an actual and potential cause of strangulated intraabdominal hernia. Surg Gynecol Obstet 1934;1934:906-13.

3. Bernal Eusse A, Restrepo Molina R, Bernal Cuartas C, Castaño Llano R. Two cases of congenital foramina in the broad ligament of the uterus with small bowel hernias and reversible intestinal distress. Rev Col Gastroenterol 2012;27(1):52-7.

4. Gray TW, Baillie DM. Hernia of the ovary and fallopian tube into the broad ligament. Can Med Assoc J 1933;29(6):647-8.

5. Allen WM, Masters WH. Traumatic laceration of uterine support; the clinical syndrome and the operative treatment. Am J Obstet Gynecol 1955;70(3):500-13.

6. Simstein NL. Internal herniation through a defect in the broad ligament. Am Surg 1987;53(5):258-9.
7. Chatman DL. Pelvic peritoneal defects and endometriosis: Allen-Masters syndrome revisited. Fertil Steril 1981;36(6):751-6.

8. Haas D, Oppelt $P$, Shebl O, Chvatal R, Shamiyeh A, Mayer R, et al. Allen-Masters syndrome: do the classic risk factors also apply in patients with endometriosis? J Obstet Gynaecol Res 2013;39(11):1513-7.

9. Batt RE. Allen-Masters syndrome is caused by trauma, not by endometriosis. J Am Assoc Gynecol Laparosc 1995;2(2):245-7.

10. Karmali S, Zurawin RK, Sherman V. Herniation through the broad ligament. CMAJ 2010;182(2):174.

11. Ferrer-Marquez M, Rico-Morales Mdel M, CarviaPousaille C, Maturana-lbanez V, Belda-Lozano R. [Acute abdomen due to Allen-Masters syndrome. An unusual finding]. Cir Esp 2008;84(6):344-5.

12. Gaster J. Internal hernia with strangulation of bowel due to a defect in the falciform ligament. Ann Surg 1948;128(2):248-52.

13. Gupta N, Boler L, Blankstein J. Bowel herniation through broad ligament mimicking ovarian torsion: a novel presentation. J Clinical Gynecology and Obstetrics 2013;2(2):93-5.

14. Lewis M. Small-bowel obstruction secondary to traumatic lacerations of the uterine supports (The Allen-Masters syndrome). J Dis Colon Rectum 1969;12(4):253-5.

15. Varela GG, López-Loredo A, León JFG. Broad ligament hernia-associated bowel obstruction. JSLS 2007;11(1):127-30.

16. Bates GJ, Bennett IC, Furnival CM, Gough IR. A strangulated hernia through the broad ligament causing ureteric obstruction. J R Coll Surg Edinb 1983;28(5):335.

17. Demir H, Scoccia B. Internal herniation of adnexa through a defect of the broad ligament: case report and literature review. J Minim Invasive Gynecol 2010;17(1):110-2.

18. Kyosola K. Simultaneous occurrence of ovarian torsion and gangrenous strangulation through a congenital opening in the mesosalpinx. Ann Chir Gynaecol 1977; 66(6): 290-1.

19. Masselli G, Derchi L, McHugo J, Rockall A, Vock P, Weston $M$, et al. Acute abdominal and pelvic pain in pregnancy: ESUR recommendations. Eur Radiol 2013;23(12):3485-500.

20. Miller DL. Safety assurance in obstetrical ultrasound. Semin Ultrasound CT MR 2008;29(2):156-64. 